\title{
Quantitative Differences in Biosynthesis and Extracellular Deposition of Fibrillin in Cultured Fibroblasts Distinguish Five Groups of Marfan Syndrome Patients and Suggest Distinct Pathogenetic Mechanisms
}

\author{
Takeshi Aoyama, * Uta Francke, ${ }^{\text {\$\$ }}$ " Harry C. Dietz,' and Heinz Furthmayr* \\ *Departments of Pathology, ${ }^{\ddagger}$ Genetics, ${ }^{8}$ Pediatrics, "Howard Hughes Medical Institute, Stanford University, Stanford, California 94305 ; \\ and 'Department of Pediatrics, The Johns Hopkins University School of Medicine, Baltimore, Maryland 21205
}

\begin{abstract}
Pulse-chase studies of $\left[{ }^{35} \mathrm{~S}\right]$ cysteine-labeled fibrillin were performed on fibroblast strains from 55 patients with Marfan syndrome (MFS), including 13 with identified mutations in the fibrillin-1 gene and 10 controls. Quantitation of the soluble intracellular and insoluble extracellular fibrillin allowed discrimination of five groups. Groups $I(n=8)$ and II $(\boldsymbol{n}=19)$ synthesize reduced amounts of normal-sized fibrillin, while synthesis is normal in groups III $(n=6)$, IV $(n=18)$, and V $(n=4)$. When extracellular fibrillin deposition is measured, groups I and III deposit between 35 and $70 \%$ of control values, groups II and IV $<35 \%$, and group $\mathrm{V}>\mathbf{7 0 \%}$.

A deletion mutant with a low transcript level from the mutant allele and seven additional patients have the group I protein phenotype. Disease in these patients is caused by a reduction in microfibrils associated with either a null allele, an unstable transcript, or an altered fibrillin product synthesized in low amounts. In 68\% of the MFS individuals (groups II and IV), a dominant negative effect is invoked as the main pathogenetic mechanism. Products made by the mutant allele in these fibroblasts are proposed to interfere with microfibril formation. Insertion, deletion, and exon skipping mutations, resulting in smaller fibrillin products, exhibit the group II phenotype. A truncated form of fibrillin of $60 \mathrm{kD}$ was identified with specific fibrillin antibodies in one of the group II cell culture media. Seven of the nine known missense mutations, giving rise to abnormal, but normal-sized fibrillin molecules, are in group IV. (J. Clin. Invest. 1994. 94:130-137.) Key words: fibrillin biosynthesis • dominant negative mutation $-F B N 1 \cdot$ extracellular matrix - elastin-associated microfibril
\end{abstract}

\section{Introduction}

The Marfan syndrome (MFS) ${ }^{1}$ is a systemic disorder of connective tissue and its clinical features involve primarily the ocular,

T. Aoyama's present address is Third Division, Department of Internal Medicine, Faculty of Medicine, Kyoto University, Kyoto 606, Japan. Address correspondence to H. Furthmayr, Department of Pathology, Stanford University, 300 Pasteur Drive, Stanford, CA 94305-5324.

Received for publication 14 January 1994 and in revised form 23 March 1994.

1. Abbreviation used in this paper: MFS, Marfan syndrome.

J. Clin. Invest.

(C) The American Society for Clinical Investigation, Inc. 0021-9738/94/07/0130/08 \$2.00

Volume 94, July 1994, 130-137 musculoskeletal, and cardiovascular systems (1). Several years ago, Sakai et al. (2) isolated fibrillin from the culture medium of dermal fibroblasts and provided substantial evidence for this protein to be the major component of $10-\mathrm{nm}$ microfibrils. Microfibrils are abundant in elastic and non-elastic tissues in organs that are significantly affected in patients with the Marfan syndrome. A dramatic decrease in the amount of such microfibrils has previously been demonstrated in the skin and in cultured fibroblasts from such patients (3).

Genetic linkage studies with random probes mapped the MFS locus to chromosome 15 (4). The fibrillin cDNA was cloned (5) and mapped to the same site on human chromosome 15 by in situ hybridization (6). Subsequently, genetic linkage was established between the Marfan syndrome and the fibrillin gene ( $F B N 1)$ on chromosome 15 by using intragenic markers (7). Several unique and independent missense, nonsense, and deletion mutations in $F B N 1$ have been reported, which make a strong argument for the primary involvement of this large gene in the causation of MFS (8-14). The basis for considerable intra- and interfamilial phenotypic variability remains unknown. Unique beaded microfibrils $(2,3,15)$ are the presumptive functional units that are affected. Even though the beaded microfibril presumably contains other proteins in addition to fibrillin (16), all linkage studies and mutational analyses to date point to the $F B N 1$ gene as the major locus responsible for the phenotype.

MFS is an autosomal dominant disorder in patients heterozygous for mutations at the FBN1 locus and, therefore, the normal allele is expected to produce fibrillin mRNA of normal size and amount. It is not known whether null mutations exist and cause MFS that do not produce mutant fibrillin and that allow unimpeded matrix deposition of wild-type fibrillin made from the normal allele. In contrast, a mutant allele that gives rise to defective fibrillin molecules could interfere with normal fibrillogenesis and affect the integrity of the microfibrils by a dominant negative mechanism (17), similar to that postulated for certain types of osteogenesis imperfecta (18). No direct evidence has been provided as yet, however, to prove that this mechanism plays a role in the pathogenesis of MFS. McGookey Milewicz et al. (19) have previously reported variable abnormalities in cultured fibroblasts from patients with MFS with respect to the rate of synthesis, secretion and the amount of fibrillin in the extracellular matrix. Implied pathogenetic mechanisms were not correlated with genotype. In a recent study we have determined that missense mutations involving cysteine residues in the $F B N 1$ gene result in delayed fibrillin secretion, but this may not be the most important effect of such mutations (20).

Here we report a comprehensive study of 55 fibroblast strains from clinically well characterized MFS patients by quantitative pulse-chase methods. Based on the amounts of newly synthesized cellular fibrillin and the insoluble extracellular frac- 
tion deposited during the $20-\mathrm{h}$ chase period, five cellular phenotypes were distinguished. Correlation of genotype to cellular and clinical phenotype suggests that FBN1 mutations cause disease by different pathogenetic mechanisms.

\section{Methods}

Study population. Patients ( $n=55)$, who satisfied the diagnostic criteria established by Beighton et al. (21), and 10 normal controls were selected for study from the Marfan Syndrome Clinic of the Stanford University Hospital, the Northern California Chapter of the National Marfan Association and the Johns Hopkins Medical Center (Baltimore, MD). These include nine fibroblast strains with missense mutations (20) and four with various other mutations as described recently (11, 13, 14).

Cell culture and pulse-chase labeling. Skin biopsy samples were obtained under an approved protocol from patients and controls either at cardiovascular surgery or during outpatient visits. Dermal fibroblast cultures were established in Dulbecco's modified Eagle's medium (DME) with $15 \%$ fetal calf serum (FCS). The procedures used for pulse-chase labeling, analysis by SDS-polyacrylamide gelectrophoresis (SDS-PAGE) and quantitation were done as described recently (20).

Immunoaffinity column. Monoclonal antibodies to fibrillin (mAb69 and 201 , kindly donated by Dr. L. Sakai, Oregon Health Science University, Portland, OR) were coupled to $\mathrm{CNBr}$-activated Sepharose (Pharmacia Fine Chemicals, Piscataway, NJ) as described previously (2). Cell culture medium was incubated with antibodies coupled to Sepharose for $3 \mathrm{~h}$ at room temperature. The gel was then washed extensively with $50 \mathrm{mM}$ Tris- $\mathrm{HCl}, \mathrm{pH} 7.5$, containing $0.5 \mathrm{M} \mathrm{NaCl}$ and $0.05 \%$ Tween 20. Bound material was eluted from the column with $1 \mathrm{M}$ acetic acid and immediately neutralized with $4 \mathrm{M}$ Tris, followed by dialysis against phosphate buffered saline (PBS). The eluates were analyzed by SDSPAGE as described (20).

\section{Results}

\section{Fibrillin metabolism in normal fibroblasts}

In preliminary experiments, dermal fibroblasts from control individuals were pulse labeled with $\left[{ }^{35} \mathrm{~S}\right]$ cysteine for $5,15,30$, and $60 \mathrm{~min}$. A labeling time of $30 \mathrm{~min}$ was chosen for the analysis of fibrillin (data not shown). As newly synthesized and labeled fibrillin is secreted from control fibroblasts, the amount of intracellular fibrillin is decreased by $\sim 80 \%$ at $4 \mathrm{~h}$, by $95 \%$ at $8 \mathrm{~h}$, and by $>98 \%$ at $20 \mathrm{~h}$, while it accumulates in the medium over the same period (Fig. 1). To follow the intracellular processing of fibrillin, 4- and 8-h chase periods were chosen for quantitation. About $20 \%$ of the total estimated amount of secreted fibrillin is incorporated into the extracellular matrix $8 \mathrm{~h}$ after pulsing the cells with radioactive cysteine (Fig. 1). Quantitation shows little additional deposition after $20 \mathrm{~h}$. By using conditions determined in these preliminary studies, we have examined 10 fibroblast strains from control individuals and 55 fibroblasts from patients with Marfan syndrome.

In the 10 control cell strains, the mean value for fibrillin synthesis obtained at time 0 (after $30 \mathrm{~min}$ of pulse) was defined as $100 \pm 10.8 \%$ and the normal range of fibrillin deposition at $20 \mathrm{~h}$ of chase as $100 \pm 11.8 \%$. These data were obtained by quantitating the radiolabeled fibrillin band on gels loaded with standardized amounts of radioactivity. Radioactivity counts were found to be a better denominator than numbers of cells, although similar numbers of cells were plated at the beginning of each experiment. The results obtained with normal fibroblasts validate this choice. We defined "decreased synthesis" as the production of $<70 \%$ of the normal amount of fibrillin (control

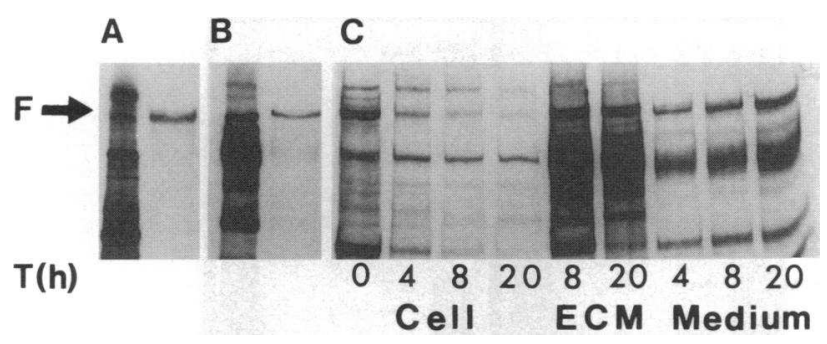

Figure 1. Synthesis, secretion, and incorporation of fibrillin into the extracellular matrix by cultured control fibroblasts. In $A$ and $B$, cells were labeled with $\left[{ }^{35} \mathrm{~S}\right]$ cysteine and fibrillin was isolated from the culture medium ( $A$, left lane) and the cell lysate $(B$, left lane) by purification using a monoclonal antibody immunoaffinity column ( $A$ and $B$, right lanes) and samples were analyzed by SDS-PAGE and autoradiography. In $C$, cell lysate ( $\mathrm{Cell}$ ), NP-40 insoluble matrix components $(E C M)$ and tissue culture medium (Medium) of control fibroblasts are shown that were pulse-labeled for $30 \mathrm{~min}$ and chased for 4,8 , or $20 \mathrm{~h}$ $(\mathrm{T}[\mathrm{h}])$. Most of the newly synthesized and labeled fibrillin $(F)$, collected after the 30 -min pulse at time 0 , is secreted from the cells during the 4-8-h chase period. It accumulates in the medium during that time and a fraction of the labeled molecules is incorporated into the extracellular matrix within 8-20 h. Fibrillin was quantitated in 30-min cell lysates (synthesis) and $20 \mathrm{~h} \mathrm{ECM} \mathrm{(deposition)} \mathrm{and} \mathrm{not} \mathrm{in} \mathrm{the} \mathrm{media,}$ because the latter showed too much variability (not shown).

value \pm 2 SD, Table I; see data presented below in Figs. 2 and 3 A). Likewise, "decreased deposition" was defined as the amount of fibrillin incorporated into the extracellular matrix being $<70 \%$ of controls (control value $\pm 2 \mathrm{SD}$, Table I; compare also Figs. 2 and $3 \mathrm{~B}$ ). In control fibroblasts, the amount of intracellular fibrillin after a 4-h chase was $18 \pm 4.8 \%$ compared with that at time 0 . When $>30 \%$ of fibrillin was retained inside the cells at the 4-h time point, we defined this as "delayed secretion" (20). Cells displaying this altered secretion pattern were found in groups III and IV (see data below in Fig. 2, $C^{\prime}$ and $D^{\prime}$ ).

\section{Fibrillin metabolism in MFS fibroblasts}

Based on the amounts of fibrillin synthesized and incorporated into the extracellular matrix, the 55 patients could be divided into five groups (Table 1).

Group I: Decreased synthesis and normal fibrillin deposition. Fibroblasts of this group $(n=8)$ synthesize about half the amount of the $350-\mathrm{kD}$ fibrillin polypeptide as compared with controls (Table I, Figs. $2 A$ and $3 A$ ), and its secretion occurs at a normal rate. Likewise, only about half the amount

Table I. Definition of Fibrillin Phenotypic Groups and Distribution of MFS Patients

\begin{tabular}{lcccccc}
\hline \multicolumn{1}{c}{ Group } & I & II & III & IV & V \\
\hline Synthesis* & $<70 \%$ & $<70 \%$ & $\geq 70 \%$ & $\geq 70 \%$ & $\geq 70 \%$ \\
$\begin{array}{l}\text { Deposition* } \\
\begin{array}{l}\text { Number of patients } \\
\text { (total = 55) }\end{array}\end{array}$ & 8 & 19 & 6 & 18 & 4 \\
\hline
\end{tabular}

* The amount of fibrillin synthesized or incorporated into the extracellular matrix was compared with that of control fibroblasts (defined as $100 \%$ ) in each experiment. 


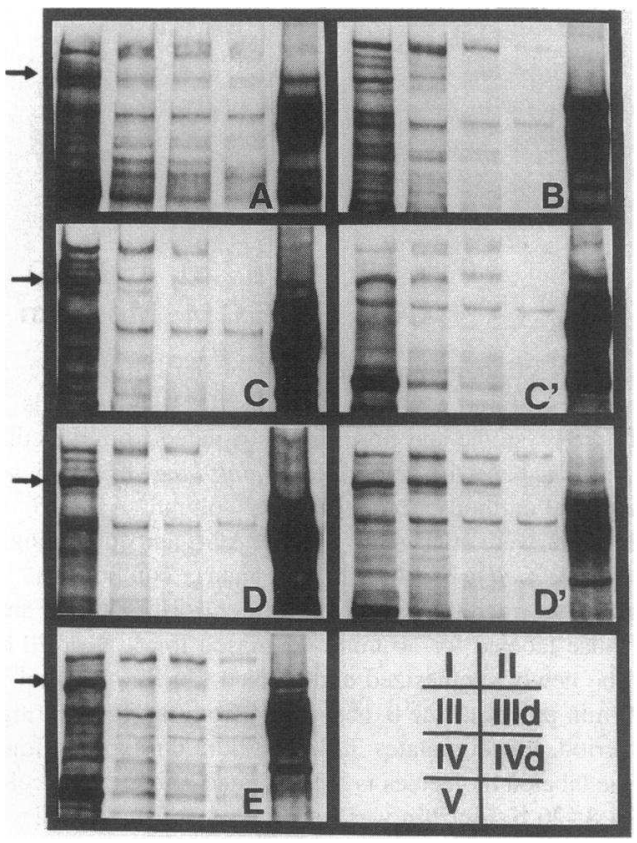

Figure 2. Synthesis, secretion and incorporation of fibrillin into the extracellular matrix by cultured fibroblasts from MFS individuals representing seven different phenotypic groups. The group identification numbers are indicated in the bottom right panel (see also Table I). Each panel consists of five lanes. Cells were labeled for $30 \mathrm{~min}$ and cell extracts at $0,4,8$, and $20 \mathrm{~h}$ (lanes 1-4) and the 20-h insoluble matrix (ECM, lanes 5) were analyzed by SDS-PAGE and autoradiography. Arrows indicate the position of the fibrillin polypeptide. Note the near absence of fibrillin from the extracellular matrix in groups II $(B)$, IV $(D)$, and $\operatorname{IVd}\left(D^{\prime}\right)$. Quantitation is required to substantiate the decrease in synthesis in groups I $(A)$ and II $(B)$ to approximately half of normal, and the reduction in deposition in group III $(C)$. The bottom left panel $(E)$ is an example of group $\mathrm{V}$, which is indistinguishable from control cells. For a definition of groups I through V, see Table I. In groups IIId and IVd $>30 \%$ of synthesized fibrillin in comparison to controls are still present in cells after a 4-h chase period (lanes 3 ). This phenotype has been designated $d$ for having delayed intracellular transport or secretion (20).

of fibrillin, as compared with that of normal controls, is incorporated into the extracellular matrix (Fig. $2 A$ and $3 B$ ). Although overall fibrillin synthesis is decreased, the matrix deposition of the presumptive normal fibrillin molecules, which account for $50 \%$ of normal, seems to be little or not at all affected in this group of patients.

Group II: Decreased synthesis and impaired fibrillin deposition. In fibroblasts of group II $(n=19)$, approximately half the normal amount of $350-\mathrm{kD}$ fibrillin polypeptides is synthesized and is secreted without delay (Table I, Figs. $2 B$ and $3 A$ ). In contrast to group I cells, however, the amounts of fibrillin recovered in the insoluble fractions are moderately to severely reduced in the range from 5 to $35 \%$ of normal (Figs. $2 B$ and $3 B$ ).

Group III: Normal synthesis of fibrillin and mildly impaired fibrillin deposition. In group III $(n=6)$, normal amounts of fibrillin are made (Table I, Figs. $2 C$ and $3 A$ ) and $>35 \%$ (35$70 \%$ ) of the synthesized fibrillin molecules are incorporated into the extracellular matrix (Fig. $3 B$ ). Fibroblasts from two of the six patients in this group showed a delay in secretion as defined above (Fig. $2 C^{\prime}$ ).
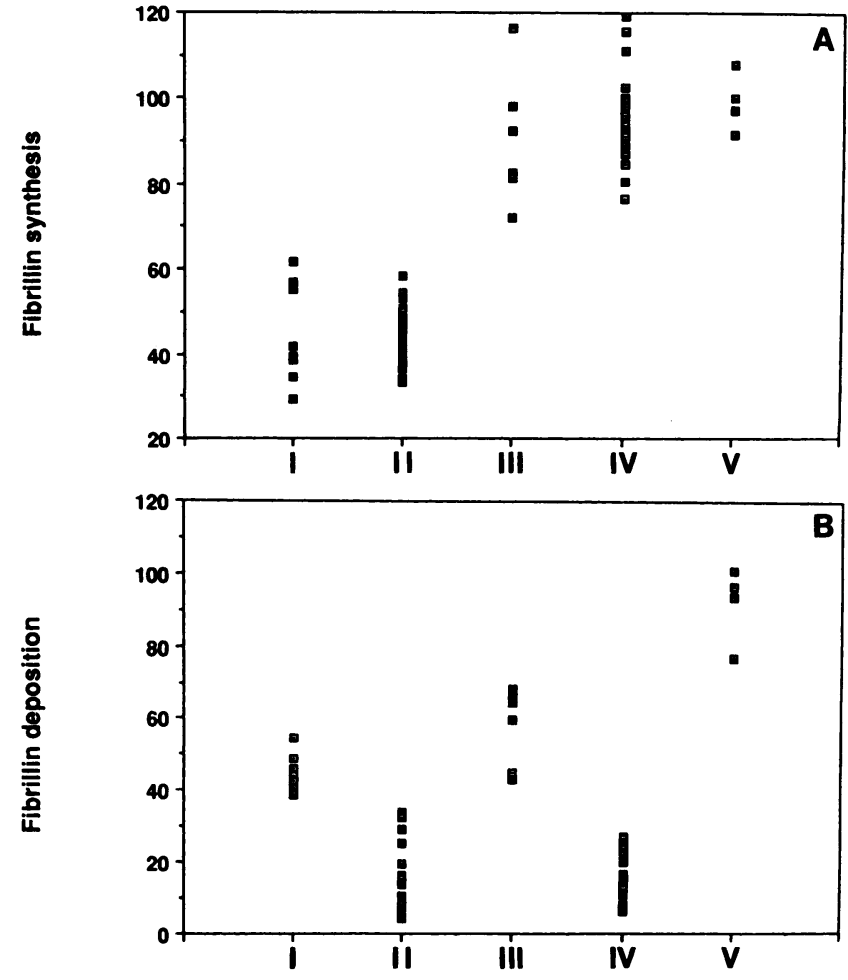

Figure 3. Fibrillin synthesis and deposition. In $A$, the amounts of newly synthesized fibrillin in cells after the 30 -min pulse as compared to that of controls are given for the five groups. In group I and II, this varies from 30 to $65 \%$ in comparison to controls. In groups III, IV, and V, the amounts of fibrillin range from 75 to $120 \%$ as compared to controls. Control cells generated values of $100 \pm 10.8 \%$. In $B$, the incorporation of fibrillin into the extracellular matrix (ECM) is given for each group. The amount of fibrillin in the ECM is $35-70 \%$ in groups I and III, and $<35 \%$ in groups II and IV. Group V is indistinguishable from controls.

Group IV: Normal synthesis and severely impaired deposition of fibrillin. Similar to group III, fibroblasts of group IV ( $n$ $=18$ ) generate normal amounts of the $350-\mathrm{kD}$ fibrillin polypeptide (Table I, Figs. $2 D$ and $3 A$ ). In six of the 18 cell strains, however, fibrillin secretion was found to be delayed (Fig. 2 $\left.D^{\prime}\right)$. In contrast to group III and regardless of the secretion phenotype, the amount of fibrillin in the matrix is drastically reduced to $<35 \%$ of normal in all of the group IV fibroblasts (Fig. $3 \mathrm{~B}$ ).

Group V: Normal synthesis, secretion, and deposition of fibrillin. In fibroblast strains of four of the patients no significant difference from controls was observed in either synthesis, secretion, or deposition of fibrillin (Figs. $2 E$ and $3, A$ and $B$ ). Studies were repeated on different passage fibroblasts and these results were consistent.

Thus our studies revealed phenotypic differences in fibrillin synthesis and/or matrix deposition in 51 of 55 (93\%) of fibroblast cultures from MFS patients.

\section{Pathogenetic mechanisms}

To examine whether correlations exist with specific pathogenetic mechanisms, we carried out the following in vitro experiments.

Group I: "Product effect" in microfibrillar assembly. The finding of cell strains that produce half the normal amount of 


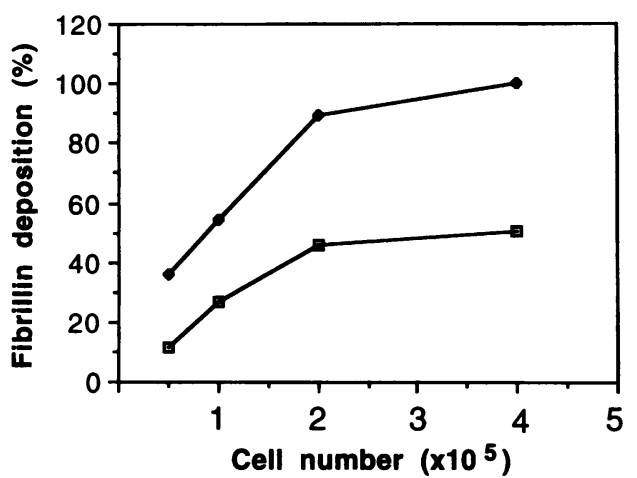

Figure 4. Effect of reduced fibrillin biosynthesis on microfibril deposition ("Product effect"). Various cell numbers of group I ( $\square$ ) and control fibroblasts (filled squares) were plated ranging from $5 \times 10^{4}$ to $4 \times 10^{5} /$ dish and fibrillin deposition was measured in comparison to standard numbers of control cells. Fibrillin deposition increased as a function of cell number at similar rates and a plateau was reached in both fibroblast strains. Fibrillin deposition of group I cells, however, was always half the amount of control values.

fibrillin allowed us to investigate the effects of cell density and of intra- and extracellular fibrillin concentration on the synthesis and deposition of fibrillin. We plated different numbers of fibroblasts from a group I patient and from a control individual. The cells were labeled as described, and after a 20-h chase period, the NP-40-insoluble extracellular components were harvested. In control fibroblasts, the incorporation of fibrillin into the extracellular matrix increases with increasing cell numbers and reaches a plateau (Fig. 4). In the group I fibroblasts, fibrillin deposition also increases with cell number, but the amount of newly deposited fibrillin in the extracellular matrix is only approximately half the amount of that of normal fibroblasts at all the cell numbers tested. This suggests that, at least under the conditions of hyperconfluent cell culture, fibrillin expression and deposition are both constitutive, strictly gene-dosage dependent and are not subject to feedback by either concentrations of soluble fibrillin or amount of microfibrillar material. To indicate that in group I fibroblasts the lower amount of insoluble fibrillin is due to lower fibrillin production, we have called this "product effect." Fibrillin appears to be deposited in the extracellular matrix with similar efficiency even in cells that make fewer normal fibrillin molecules. The observed reduced deposition in group I cells could thus simply be due to fewer available fibrillin molecules.

Groups II and IV: Interference by abnormal fibrillin molecules with normal microfibrillar assembly ("Medium exchange"'). In groups II and IV, $<35 \%$ of de novo synthesized fibrillin is deposited in the extracellular matrix compared to $100 \pm 10 \%$ in controls (Table $I$ and Figs. 2 and 3). This result could be explained if the mutant allele gives rise to abnormal protein molecules that interfere with fibrillogenesis. Under this hypothesis, group II fibroblasts are expected to make normal fibrillin (the product of the normal allele) in addition to a truncated abnormal fibrillin polypeptide, which we would not have detected in the pulse-chase experiments. Group IV fibroblasts, on the other hand, would make both normal and full-size, but altered, fibrillin molecules. In both instances, the abnormal protein product might affect microfibrillar assembly.

To test this hypothesis, we carried out "medium exchange",

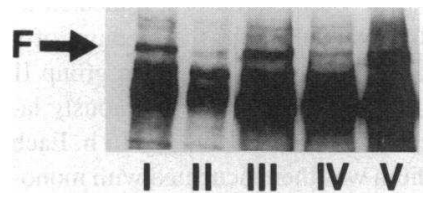

Figure 5. Interference in matrix deposition of labeled normal fibrillin by MFS group II and IV cells ("Medium exchange"). Control fibroblasts were pulsed for $30 \mathrm{~min}$ and chased for $16 \mathrm{~h}$. Labeled medium was transfered to dishes containing unlabeled fibroblasts from each of the five MFS groups. After $20 \mathrm{~h}$ incubation, the NP-40-insoluble material was analyzed by SDS-PAGE. The amount of labeled fibrillin is dramatically reduced in the matrix of the group II and group IV cells, but not of those of groups I, III, and V.

experiments. Fibroblasts of all five groups were pulse labeled for $30 \mathrm{~min}$ and then chased for $16 \mathrm{~h}$. When labeled medium was transferred to dishes containing normal unlabeled fibroblasts, labeled fibrillin (derived from the MFS cells) could be recovered from the extracellular matrix of control cells, but no differences between the groups were observed (data not shown). This suggests that labeled fibrillin molecules made by the MFS cells are incorporated into the extracellular matrix. It suggests further that under these experimental conditions small amounts of abnormal products made by the MFS cells during the 30min pulse (product of the mutated allele) have no detectable effect on fibrillin deposition. We, therefore, did the opposite experiment.

Medium containing labeled fibrillin collected from control fibroblasts after $16 \mathrm{~h}$ of continuous labeling was transferred to dishes containing unlabeled fibroblasts from all the different MFS groups. After an additional 20-h incubation, the NP-40 insoluble matrix was harvested and analyzed. As shown in Fig. 5 , the incorporation of labeled normal fibrillin molecules into the extracellular matrix of unlabeled MFS fibroblasts, which continue to make fibrillin products during the 20-h incubation, was considerably reduced for groups II and IV in contrast to cells from groups I, III, or V. These results resemble the data presented above on the direct analysis of fibrillin deposition in MFS from the various groups (Fig. 2). It suggests either interference with matrix deposition by an abnormal fibrillin product, as discussed above, or less efficient "binding"' of normal fibrillin molecules under medium exchange conditions to a MFS matrix of group II and IV cells that, by definition, contains less insoluble fibrillin.

Purification of a truncated form of fibrillin from group II fibroblasts. To determine, whether the media of group I and II MFS fibroblasts contain truncated fibrillin molecules, we labeled group II ( $n=15)$, group I $(n=3)$, and control fibroblasts $(n=2)$ continuously for $16 \mathrm{~h}$ with $50 \mu \mathrm{Ci} / 700 \mu \mathrm{l}$ of $\left[{ }^{35} \mathrm{~S}\right]-$ cysteine. The culture medium of each cell strain was then passed over a fibrillin antibody immunoaffinity column and the eluates were analyzed by both 4.5 and $10 \%$ SDS-PAGE. While we were able to isolate a single $350-\mathrm{kD}$ fibrillin polypeptide species from all of the cells, the eluate from the mab69 antibody column of one of the group II fibroblasts contained two labeled bands, one at $350 \mathrm{kD}$ and the other at $\sim 60 \mathrm{kD}$ (Fig. 6). The $60 \mathrm{kD}$ polypeptide was not seen in eluates from control cells or any of the other MFS fibroblasts and, thus, does not seem to be an intra- or extracellularly processed form or degradation product of fibrillin. The $60-\mathrm{kD}$ band most likely represents a truncated fibrillin molecule due to an as yet unidentified $F B N 1$ mutation. From the intensity of the radioactive band we estimate that the amount of the "short" fibrillin product could correspond to 


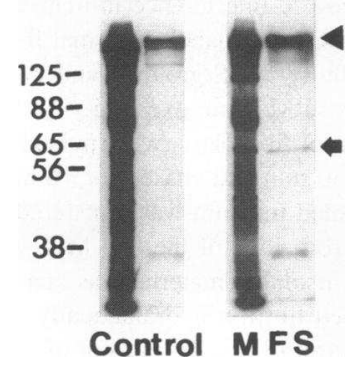

Figure 6. Purification of a truncated fibrillin molecule from the medium of a group II fibroblast strain. The group II and control cells were continuously labeled with $\left[{ }^{35} \mathrm{~S}\right]$ cysteine for $16 \mathrm{~h}$. Each medium was then incubated with monoclonal antibody coupled to $\mathrm{CNBr}$-activated Sepharose. Bound material was eluted and analyzed by $10 \%$ SDS-

PAGE. In addition to normal-sized fibrillin (arrowhead), this particular group II fibroblast strain synthesized an additional $60-\mathrm{kD}$ peptide (arrow) that

was not observed in any of the other cell strains. The $35-\mathrm{kD}$ fragment of unknown identity (not marked) is present in all preparations.

$\sim 50 \%$ of normal fibrillin, considering that it is only one fifth the normal size.

\section{Discussion}

The discovery of different types of mutations in the $F B N 1$ gene have proven the causal relationship between fibrillin abnormalities and the autosomal dominant Marfan syndrome (8-14). Previously, cells from several MFS patients were shown to either produce shortened fibrillin molecules (10), to produce less, to deposit less, or to secrete fibrillin more slowly (19). In addition to these latter three alterations in biosynthesis, the existence of a fibrillin precursor was postulated based on a difference in molecular weight between the intra- and extracellular form (19). The latter observation has not been confirmed in other studies $(15,20)$, nor did we find evidence for profibrillin here. In our preliminary studies we experienced difficulties in making assignments to the three basic groups described by McGookey Milewicz et al. (19). By applying quantitative pulse-chase labeling of fibroblasts we have found now that patients diagnosed with MFS can be subdivided into five groups. Since four of these five groups contain samples with known FBN1 mutations, correlations between protein phenotype and pathogenetic mechanisms could also be made.

Fibroblasts in groups I and II account for $\sim 50 \%$ of the MFS samples studied here. These cells make half the amount of fibrillin as compared with controls. The two groups are distinguished by the amount of fibrillin that becomes insoluble during the $20-\mathrm{h}$ chase period. While the group I phenotype shows one half of normal deposition, this value is even lower in group II. The major difference between these two groups appears to be related to the amount of mutant transcript and protein derived from the mutant allele. Evidence for this comes from four patients with known FBNI mutations (13; Table II). As has been observed also for other disease genes $(23,24)$, certain mutations affect transcript levels. In one group I patient, an insertion of four nucleotides leads to a frameshift and premature termination codon, which results in transcript levels of only $6 \%$ of normal (13). The amount of abnormal protein made by this mutant transcript has not been determined, but it is likely to be also very low. Three mutations have been identified in group II patients. Two of them, -2448 (247)G +1 to $A$ and 770 (3464)del17, create frameshifts and premature signals for termination of translation of mRNA and both result in transcript levels of $\sim 15 \%$ of normal $(13,14)$. The third, 3645(6339)T to $\mathrm{G}$, a nonsense mutation that leads to in-frame skipping of exon 51 (11), generates $25 \%$ of mutant mRNA. The amount of transcript in the three mutations of group II are higher as compared with group I, suggesting that a corresponding larger amount of altered protein in group II may be responsible for the significantly lower deposition of fibrillin in this group (13; Table II).

Our experiments on the "product effect" show that a decrease in synthesis and/or deposition of fibrillin does not seem to lead to compensatory changes in fibrillin metabolism. Thus, a low amount or total lack of mutant product would result in a group I and possibly mild disease phenotype due to a $50 \%$ decrease of extracellular fibrillin and an overall reduction in microfibrils (Fig. 7). This would be analogous to cases of osteogenesis imperfecta with a nonfunctional allele for pro- $\alpha 1$ (I) chains of type I collagen (25).

Altered fibrillin products in group II fibroblasts, however, seem to act as competitive inhibitors of microfibrillar assembly and result in $<35 \%$ deposition. A plausible explanation for the pathogenetic effect of the documented mutations that lead to truncated proteins is based on a "dominant negative" mechanism. As shown for a number of other disorders $(17,18,26$, 27 ), the abnormal product of the mutant allele interferes with

Table II. Known FBN1 Mutations and Their Protein Phenotypes

\begin{tabular}{|c|c|c|c|c|c|c|}
\hline Mutation & Type & Synthesis & Deposition & mRNA & Group & Reference \\
\hline 2444 (5138)ins4 & frameshift & 56.7 & 54.3 & 6 & I & 13 \\
\hline$-2448(247)$ & nonsense & 50.7 & 25.1 & 16 & II & 13 \\
\hline 770 (3464)del17 & frameshift & 42.3 & 6.6 & 15 & II & 14 \\
\hline 3645 (6339)T to $G$ & exon skip & 58.4 & 8.9 & 25 & II & 11 \\
\hline 2072 (4766)G to $T$ & missense & 81.7 & 64.5 & - & III & 14,20 \\
\hline 2293 (4987)T to C & missense & 92.5 & 44.9 & - & III & 34,20 \\
\hline $656(3350) \mathrm{G}$ to $\mathrm{A}$ & missense & 100 & 24.3 & - & IV & 14,20 \\
\hline$-111(2584) \mathrm{T}$ to $\mathrm{C}$ & missense & 111 & 13.1 & - & IV & 14,20 \\
\hline $716(3410) \mathrm{G}$ to $\mathrm{C}$ & missense & 84.9 & 13.3 & - & IV & 8,20 \\
\hline $4226(6920) G$ to $C$ & missense & 98 & 21.1 & - & IV & 9,20 \\
\hline 1052 (3746)G to $C$ & missense & 115.7 & 27.4 & - & IV & 34,20 \\
\hline 3968 (6662)G to $C$ & missense & 94.3 & 26.6 & - & IV & 34,20 \\
\hline-1052 (1643)A to $T$ & missense & 96.1 & 22.8 & - & IV & 13,20 \\
\hline
\end{tabular}




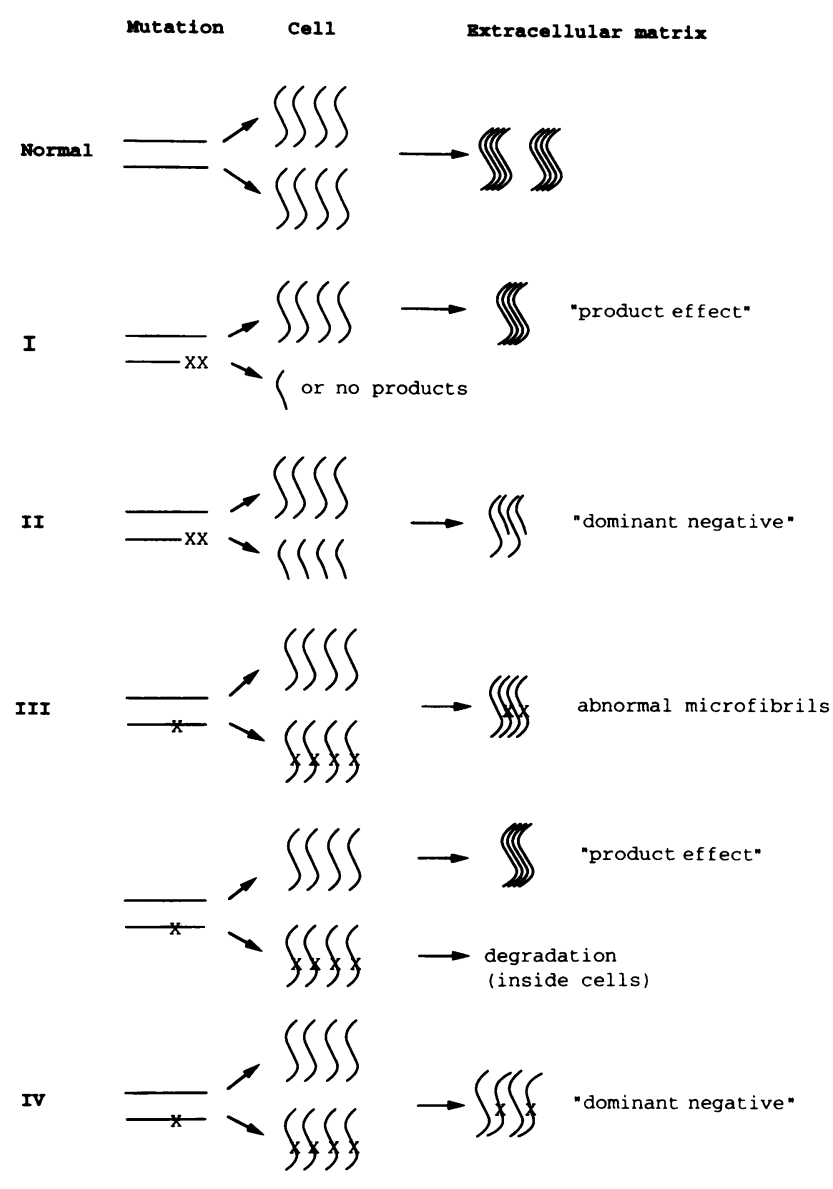

Figure 7. Schematic representation of postulated pathogenetic mechanisms in MFS. In control cells, the products of both alleles are normal fibrillin molecules that are assembled into microfibrils extracellularly. Group I cells make fibrillin from the normal allele and either fail to synthesize any product from the mutant allele or make a (truncated) product that is not secreted or that is present in only low amounts (due to decreased transcript stability, inefficient secretion or intracellular degradation of the protein product). The normal fibrillin molecules are deposited in the extracellular matrix with normal efficiency, but fewer fibrils are formed ("product effect"). In group II, truncated fibrillin molecules are secreted and interfere with microfibril formation by a "dominant negative" mechanism. Abnormal fibrils of lower stability or very few normal fibrils are made. In group III, two possible mechanisms could be operating. One, mutations causing minimal effects on fibrillin structure could lead to microfibrils formed from both normal and mutant fibrillin molecules, or two, similar to group I, mutant fibrillin molecules are degraded and reduced amounts of normal microfibrils are deposited. In group IV, mutant fibrillin molecules of normal size are secreted and interfere with fibrillogenesis by a "dominant negative" mechanism.

the function of the normal protein, the product of the normal allele. To obtain experimental evidence, we have attempted to interfere with fibrillin deposition by "medium exchange", experiments and to isolate abnormal fibrillin products with antibodies. The former experiment yielded satisfying results by demonstrating that low levels of fibrillin deposition are only seen in group II and IV fibroblasts and not in the other three groups. This low fibrillin deposition could be due to a true dominant negative effect, namely inhibition by abnormal fibrillin products. It could also be related to a large reduction in "binding sites" for the labeled fibrillin molecules in the extracellular matrix, since the cell matrices from the two groups contain considerably lesser amounts of microfibrillar material. In a second experimental approach, we were able to obtain a shortened fibrillin molecule from one of the 15 group II patients. This low success rate of the present experiments may be due to reduced stability of the mutant protein or to inadequate antibody reagents, which may not react with the altered protein. We are pursuing various other methods and believe that, provided enough of the altered fibrillin molecules can be isolated, such molecules would be useful for studies on fibrillin assembly and for discriminating among the possibilities described above.

The "medium exchange" results, however, are of considerable interest also because they suggest that experiments of this type can be used for screening and the identification of mutations with large effects on fibrillin deposition.

Fibroblasts of group III and IV make normal amounts of $350-\mathrm{kD}$ fibrillin products that are likely to include both normal and mutant polypeptides. Fibroblasts with known FBNI missense mutations fall into these two groups (Table II). We have distinguished the two groups on the basis of the amount of extracellular fibrillin after a 20 -h chase period, which is approximately half normal in group III and significantly lower $(<35 \%)$ in group IV. The very low amounts of insoluble fibrillin in group IV fibroblasts suggests that the altered protein acts by inhibition of assembly similar to the effect postulated for the group II cells. Results of the "medium exchange" experiments also appear to support the notion that the deposition of normal fibrillin is inhibited by products of group IV, but not III cells (Fig. 6), but do not exclude other possibilities. The medium exchange experiments argue against the formation of abnormal and unstable microfibrils that are rapidly degraded. Additional experiments will be required, however, to formally exclude this and other possibilities.

Other effects of FBNI missense mutations in groups III and IV include delay in intracellular transport and secretion (13, 20 ), which could have a modulating influence on the extracellular microfibrillar assembly process. Fibroblasts from 6 of 18 patients in group IV, and 2 of 6 in group III showed this delay in secretion, presumably because of abnormal intracellular transport of the mutant protein (20).

Group III appears to be more heterogeneous with respect to phenotype and pathogenetic mechanisms. In one group III fibroblast strain the intracellular transport of fibrillin was delayed and it was degraded intracellularly (data not shown, Fig. 7). Such a mutation might yield a phenotype more akin to the one described for group I cells above, since no mutant protein is found in the extracellular matrix. Possible explanations for other cell strains include incorporation of mutant molecules into the extracellular matrix and, because of lower efficiency of interaction with other molecules, one might expect somewhat lower amounts of insoluble fibrillin than in control cells. Such microfibrils, however, could conceivably be structurally or functionally abnormal (Fig. 7).

In four of the 55 fibroblast strains no defect in fibrillin metabolism could be identified with the pulse-chase method. All four were sporadic cases and, therefore, linkage studies with polymorphic markers from the $F B N 1$ locus were not possible. We are considering two possibilities to explain this finding. Firstly, some proteins, such as elastin (28), thrombospondin (29), microfibril associated glycoprotein (MAGP; 30), emilin (31), associated microfibril protein (32), and FBN2 (33) have 
been reported to be associated with microfibrils. Chromosomal localizations of genes for these proteins are different from the $F B N 1$ locus. $F B N 2$ has been linked to a distinct phenotype, namely congenital contractural arachnodactyly (33). Although linkage evidence for genetic heterogeneity in the Marfan syndrome has not been reported to date, alteration of the structure or expression of these proteins or other, as yet unknown proteins associated with microfibrils, could affect the microfibril functionally and could cause clinical features similar to those seen in MFS. Secondly, in these four patients we have only examined the amount of insoluble fibrillin. It is possible that in group V, the defect is not seen as an abnormality in amount, but rather in quality or function of the microfibrils.

We have found defects in fibrillin metabolism of cultured fibroblasts in $93 \%$ of the patients with MFS by this quantitative pulse-chase method. This success rate contrasts with the relatively small fraction of patients for whom mutations have been defined at the mRNA level using denaturing gradient gel electrophoresis or single strand conformation analysis of RT-PCR products and direct sequencing of such products (14). With one exception (8), mutations in each sporadic case or family with MFS have been different.

Although little is known about microfibrillar assembly, we propose that the fibrillin molecules deposited in short term tissue culture can provide a measure for fibrillin's assembly into microfibrils. Conceivably, ${ }^{35} \mathrm{~S}$-labeled fibrillin molecules are interacting with already formed microfibrils in the matrix. Microfibril formation is dependent on the amount of mutant transcript, the efficiency of intracellular processing, and the stability and conformational changes of the mutant protein. The amount of fibrillin in the extracellular matrix, as determined by pulsechase methods, appears to reflect a number of these factors. The classification of patients into the five groups may also have the additional advantage of providing a basis for analyzing the relationship between clinical phenotype and underlying pathogenesis, which is currently being undertaken (Aoyama et al., in preparation). The method we have used in this study also promise to provide a diagnostic tool, which will be quite helpful for the genetic counseling and management of patients with Marfan syndrome or related disorders.

\section{Acknowledgments}

We thank the medical staff of the Marfan Syndrome Clinic at Stanford University for generous support, Craig Miller for tissue samples, Cheryll Gasner for collection of samples and clinical data, and Petra Wilgenbus and Erika Valero for expert technical assistance.

This study was supported by the Howard Hughes Medical Institute (U. Francke), National Institutes of Health research grants ( $H$. Furthmayr and H. Dietz), and fellowship support from the Kyle-Mann Research Fund and the Japanese Heart Association (T. Aoyama).

\section{References}

1. Pyeritz, E. R., and V. A. McKusick. 1979. The Marfan syndrome: diagnosis and management. N. Engl. J. Med. 300:772-777.

2. Sakai, L. Y., D. R. Keene, and E. Engvall. 1986. Fibrillin, a new 350-kD glycoprotein, is a component of extracellular microfibrils. J. Cell Biol. 103:24992509.

3. Hollister, D. W., M. Godfrey, L. Sakai, and R. E. Pyeritz. 1990. Immunohistologic abnormalities of the microfibrillar-fiber system in the Marfan syndrome. N. Engl. J. Med. 323:152-159.

4. Kainulainen, K., L. Pulkkinen, A. Savolainen, I. Kaitila, and L. Peltonen. 1990. Location on chromosome 15 of the gene defect causing Marfan syndrome. N. Engl. J. Med. 323:935-939.
5. Maslen, C. L., G. M. Corson, B. K. Maddox, R. W. Glanville, and L. Y. Sakai. 1991. Partial sequence of a candidate gene for the Marfan syndrome. Nature (Lond.). 352:334-337.

6. Magenis, R. E., C. L. Maslen, L. Smith, L. Allen, and L. Sakai. 1991. Localization of the fibrillin gene to chromosome 15, band 15q21.1. Genomics. 11:346-351.

7. Tsipouras, P., R. D. Mastro, M. Sarfarazi, B. Lee, E. Vitale, A. H. Child, M. Godfrey, R. B. Devereux, D. Hewett, B. Stenmann, D. Viljoen, B. C. Sykes, M. Kilpatric, F. Ramirez and the international Marfan Syndrome collaborative study. 1992. Genetic linkage of the Marfan syndrome, ectopia lentis, and congenital contractural arachnodactyly to the fibrillin genes on chromosome 15 and 5. N. Engl. J. Med. 326:905-909.

8. Dietz, H. C., G. R. Cutting, R. E. Pyeritz, C. L. Maslen, L. Y. Sakai, G. M. Corson, E. G. Puffenberger, A. Hamosh, E. J. Nanthakumar, S. M. Curristin, G. Stetten, D. A. Meyers, and C. A. Francomano. 1991. Marfan syndrome caused by a recurrent de novo missense mutation in the fibrillin gene. Nature (Lond.). 352:337-339.

9. Dietz, H. C., R. E. Pyeritz, E. G. Puffenberger, R. J. Kendzior Jr, G. M. Corson, C. L. Maslen, L. Y. Sakai, C. A. Francomano, and G. R. Cutting. 1992. Marfan phenotype variability in a family segregating a missense mutation in the epidermal growth factor-like motif of the fibrillin gene. J. Clin. Invest. 89:16741680.

10. Kainulainen, K., L. Y. Sakai, A. Child, F. M. Pope, L. Puhakka, L. Ryhanen, A. Palotie, L. Kaitila, and L. Peltonen. 1992. Two mutations in Marfan syndrome resulting in truncated fibrillin polypeptides. Proc. Natl. Acad. Sci. USA. 89:5917-5921.

11. Dietz, H. C., D. Valle, C. A. Francomano, R. J. Kendzior, R. E. Pyeritz, and G. R. Cutting. 1993. The skipping of constitutive exons in vivo induced by nonsense mutations. Science (Wash. DC). 259:680-682.

12. Hewett, D. R., J. R. Lynch, R. Smith, and B. C. Sykes. 1993. A novel fibrillin mutation in the Marfan syndrome which could disrupt calcium binding of the epidermal growth factor-like module. Hum. Mol. Genet. 2:475-477.

13. Dietz, H. C., I. McIntosh, L. Y. Sakai, G. M. Corson, S. C. Calberg, R. E. Pyeritz, and C. A. Francomano. 1993. Four novel FBN1 mutations: significance for mutant transcript level and EGF-like domain calcium binding in the pathogenesis of Marfan syndrome. Genomics. 17:468-475.

14. Tynan, K., K. Comeau, M. Pearson, P. Wilgenbus, D. Levitt, C. Gasner, M. A. Berg, D. C. Miller, and U. Francke. 1993. Mutation screening of complete fibrillin-1 coding sequence: report of five new mutations, including two in 8cysteine domains. Hum. Mol. Genet. 11:1813-1821.

15. Sakai, L. Y., D. R. Keene, R. W. Glanville, and H. P. Bachinger. 1991. Purification and partial characterization of fibrillin, a cysteine-rich structural component of connective tissue microfibrils. J. Biol. Chem. 266:14763-14770.

16. Gibson, M. A., and E. G. Cleary. 1987. The immunohistological localization of microfibril-associated glycoprotein (MAGP) in elastic and non-elastic tissues. Immunol. Cell Biol. 65:345-356.

17. Herskowitz, I. 1987. Functional inactivation of genes by dominant negative mutations. Nature (Lond.). 329:219-222.

18. Williams, C. J., and D. J. Prockop. 1983. Synthesis and processing of a type I procollagen containing shortened pro- $\alpha 1$ (I) chains by fibroblasts from a patient with osteogenesis imperfecta. J. Biol. Chem. 258:5915-5921.

19. McGookey Milewicz, M., R. E. Pyeritz, E. S. Crawford, and P. H. Byers. 1992. Marfan syndrome: defective synthesis, secretion, and extracellular matrix formation of fibrillin by cultured dermal fibroblasts. J. Clin. Invest. 89:79-86.

20. Aoyama, T., K. Tynan, H. C. Dietz, U. Francke, and H. Furthmayr. 1993. Missense mutations impair intracellular processing of fibrillin and microfibril assembly in Marfan syndrome. Hum. Mol. Genet. 12:2135-2140.

21. Beighton, P., A. Paepe, D. Danks, G. Finidori, T. Gedde-Dahl, R. Goodman, J. G. Hall, D. W. Hollister, W. Horton, V. A. McKusik, J. M. Opitz, F. M. Pope, R. E. Pyeritz, D. L. Rimoin, D. Sillence, J. W. Spranger, E. Thompson, P. Tripouras, D. Viljoen, I. Winship, and I. Young. 1988. International nosology of heritable disorder of connective tissue, Berlin, 1986. Am. J. Med. Genet. 29:581594.

22. Fleischmajer, R., P. Contard, E. Schwartz, D. MacDonald II, L. Jacobs II, and L. Sakai. 1991. Elastin-associated microfibrils $(10 \mathrm{~nm})$ in a three-dimensional fibroblast culture. J. Invest. Dermatol. 97:638-643.

23. Kadowaki, T., H. Kadowaki, M. M. Rechler, M. Serrano-Rios, J. Roth, Phillip Gorden, and S. I. Taylor. 1990. Five mutant alleles of insulin receptor gene in patients with genetic forms of insulin resistance. J. Clin. Invest. 86:254264.

24. Hamosh, A., B. J. Rosenstein, and G. R. Cutting. 1992. CFTR nonsense mutations G542X and W1282X associated with severe reduction of CFTR mRNA in nasal epithelial cells. Hum. Mol. Genet. 1:542-544.

25. Barsh, G. S., K. E. David, and P. H. Byers. 1982. Type I osteogenesis imperfecta: A nonfunctional allele for proa 1(I) chains of type I procollagen. Proc. Natl. Acad. Sci. USA. 79:3838-3842.

26. Willing, M. C., D. H. Cohn, B. Starman, K. A. Halbrook, C. R. Greenberg, and P. H. Byers. 1988. Heterozygosity for a large deletion in the $\alpha 2$ (I) collagen gene has a dramatic effect on type I collagen secretion and produces perinatal lethal osteogenesis imperfecta. J. Biol. Chem. 263:8398-8404. 
27. Vogel, B. E., R. R. Minor, M. Freund, and D. J. Prockop. 1987. A point mutation in a type I procollagen gene converts glycine 748 of the 1 chain to cysteine and destabilizes the triple helix in a lethal variant of osteogenesis imperfecta. J. Biol. Chem. 262:14737-14744.

28. Huttunen, K., I. Kaitila, A. Savolainen, A. Palotie, and L. Peltonen. 1989. The linkage analysis with RFLP markers of elastin and type III collagen genes in Finnish Marfan families. Am. J. Med. Genet. 38:244a. (Abstr.)

29. Arbeille, B. B., F. M. J. Fauvel-Lafeve, M. B. Lemesle, D., Tanza, and Y. J. Legrand. 1991. Thrombospondin: A component of microfibrils in various tissues. J. Histochem. Cytochem. 39:1367-1375.

30. Gibson, M. A., L. B. Sandberg, L. E. Grosso, and E. G. Cleary. 1991. Complementary DNA cloning establishes microfibril-associated glycoprotein (MAGP) to be a discrete component of the elastin-associated microfibrils. J. Biol. Chem. 266:7596-7601.
31. Bressan, G. M., D. Daga-Gordini, A. Colombatti, V. Marigo, and D. Volpin. 1993. Emilin, a component of elastic fibers preferentially located at the elastin-microfibrils interface. J. Cell Biol. 121:201-212.

32. Horrigan, S. K., C. B. Rich, B. W. Streeten, Z. Li, and J. A. Foster. 1992. Characterization of an associated microfibril protein through recombinant DNA techniques. J. Biol. Chem. 267:10087-10095.

33. Lee, B., M. Godfrey, E. Vitale, H. Hori, M. Mattei, M. Sarfarazi, P. Tsipouras, F. Ramirez, and D. W. Hollister. 1991. Linkage of Marfan syndrome and a phenotypically related disorder to two different fibrillin genes. Nature (Lond.). 352:330-334.

34. Dietz, H. C., J. M. Saraiva, R. E. Pyeritz, G. R. Cutting, and C. A. Francomano. 1992. Clustering of fibrillin (FBN1) mutations in Marfan syndrome patients at cysteine residues in EGF-like domains. Human Mutation. 1:366-374. 